\title{
SIGnOS
}

Artículos de

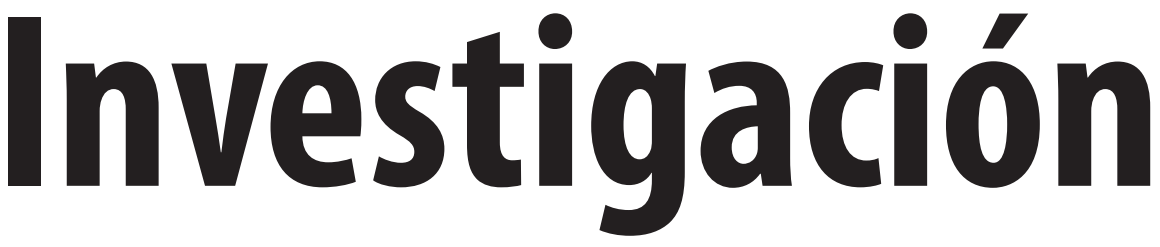





\section{Análisis de la percepción respecto al reporte de los eventos adversos y la seguridad del paciente en una IPS de alta complejidad de la ciudad de Medellín"}

\section{Analysis of perception regarding the reporting of adverse events and patient safety in a high complexity's IPS, in the city of Medellin}

Beatriz Elena Gómez Vásque: **

Convenio Universidad Santo Tomás e Icontec

\section{RESUMEN}

Una situación de riesgo en la atención y el cuidado del paciente puede conducir a la aparición de un evento adverso; este tipo de error suele ser responsabilidad, no solo de quien es agente del servicio, sino de una compleja red de fallas humanas, tecnológicas y de procesos. Así la complejidad que comprende un evento adverso no solo se puede responder con acciones aisladas y circunstanciales, por lo que para poder evitarlo es necesaria una cultura de la seguridad del paciente, dentro de las instituciones de salud. De este
Recibido: 17 de junio de 2011

Revisado: 21 de septiembre de 2011 Aceptado: 20 de octubre de 2011 


\section{ABSTRACT}

A risk, in care and patient care may result in the appearance of an adverse event. This type of error is usually the responsibility not only of the person who is a service agent, but of a complex web of human failings, technological and/or processes. Thus, the multidimensionality comprising an adverse event cannot only respond to isolated and circumstantial actions, so in order to avoid this a culture of patient safety in health institutions is necessary. Following this approach, arises the interest and the contribution of this research project which investigated the medical and paramedical staff of IPS, the third level of complexity in the city of Medellin, on the human, institutional and legal factors involved in the reporting of adverse events and knowledge of the culture of patient safety through their beliefs, attitudes and knowledge on this issue.

Keywords: Adverse events (AE), culture of patient safety, government health service providers (IPS), quality.

\section{INTRODUCCIÓN}

Con la perspectiva de la universalización del derecho a la salud, la atención en relación con este servicio se ha ubicado en el centro de debates y críticas, en cuanto a la calidad con que se presta. En este sentido, una de las principales preocupaciones es lo relacionado con la seguridad del paciente, ya que en los hospitales diariamente se producen fallas y errores en el proceso de atención en salud, tanto en los países desarrollados como en los países en vías de desarrollo, que terminan impactando de manera negativa la salud pública, en términos humanos, políticos, económicos y sociales.

Sin embargo, la buena atención a los pacientes no es un tema reciente, pues se remonta, incluso, a los dos pilares de la medicina hipocrática, como Rodríguez (2006) explica, este concepto se encuentra ya implícito en el principio de no maleficencia, "primero no hacer daño", y en el de beneficencia, "haz todo el bien que puedas". Estos dos principios evidencian la complejidad de las prácticas médicas y ponen al descubierto la necesidad de definir dos conceptos que suelen confundirse entre sí: lo que se considera un error y lo que se califica como un evento adverso (EA).

Portocarrero ${ }^{1}$ aclara la diferencia entre estos dos términos, al definir el error como una falta de planeación en la ejecución de una conducta, que puede o no causar un evento adverso. Esto significa que, para este autor, el evento adverso, en sí, es un error por acción u omisión que implica "la lesión o daño no intencional, causado por la intervención asistencial, no por la patología de base"2.

James Reason ${ }^{3}$, psicólogo inglés, añade otro elemento a esta visión al plantear que si bien el error puede ser una falla humana en completar una acción, tal como estaba contemplada, o en el uso de un plan equivocado para alcanzar un objetivo, las fallas o errores, tanto de planeación como de ejecución, están también relacionados con diferencias o contradicciones entre los sistemas que intervienen en la acción. Esta perspectiva entra en consonancia con lo expresado por la Organización Panamericana de la Salud, OPS (2005), la cual expresa que el error humano es apenas una parte del problema de las fallas en la seguridad del paciente, pues es necesario reconocer que en los procedimientos y sistemas médicos pueden existir problemas de base crónicos.

\footnotetext{
1 PORTOCARRERO. Seguridad del paciente: primero, cultura justa. En: Vía salud. 2007, no. 39, p. 4.

2 Ibíd, p. 2.

3 REASON, James, 1997, p. 126.
} 
De esta forma, los errores clínicos-asistenciales comprenden una serie de hechos diversos que incluyen "diagnósticos equivocados o demorados, fallas en la administración de medicamentos, demoras de informes de resultados, mala comunicación durante las entregas o transiciones de la atención, fallas de atención y cuidados posoperatorios inadecuados para solo mencionar algunos de los más frecuentes" ${ }^{\text {. }}$

En síntesis, estas concepciones reconocen que en el proceso de atención de salud existen diferentes situaciones calificadas como de riesgo, las cuales son consecuencia de factores humanos, tecnológicos y de procesos, y que pueden resultar o no resultar en lo que se denomina "eventos adversos".

A tono con estas preocupaciones, en Colombia, los organismos de salud han venido trabajando de una forma seria y cuidadosa en el mejoramiento de la seguridad del paciente. Así, en el 2004, en el país se impulsó el tema de los eventos adversos y se promovieron procesos de vigilancia de estos, en diferentes instituciones del país. En el mismo año, el ente acreditador en salud, el Instituto Colombiano de Normas Técnicas y Certificación (ICONTEC) estableció como condición para la acreditación que las instituciones demostraran su trabajo alrededor del tema y la implementación de procesos para su medición y vigilancia. Dos años más tarde, este proceso demostró logros importantes, debido a lo cual el Ministerio de Protección Social tomó la decisión de hacer obligatorio el cumplimiento del reporte y seguimiento de los eventos adversos en las instituciones de salud, en el territorio colombiano.

Desde esta visión, las instancias legales en Colombia consideran los EA como:

Lesiones o complicaciones involuntarias que ocurren durante la atención en salud, las cuales son más atribuibles

4 PORTOCARRERO, Op. cit., p. 3. a esta que a la enfermedad subyacente y que pueden conducir a la muerte, la incapacidad o al deterioro en el estado de salud del paciente, a la demora del alta, a la prolongación del tiempo de estancia y al incremento de los costos de la no-calidad (Resolución 1446 de 2006).

Así, para el Ministerio de la Protección Social (2008) los EA están clasificados en prevenibles y no prevenibles: el EA prevenible es el resultado no deseado, no intencional, que se habría evitado mediante el cumplimiento de los estándares del cuidado asistencial, disponibles en un momento determinado; mientras que el EA no prevenible es el resultado no deseado, no intencional, que se presenta a pesar del cumplimiento de los estándares del cuidado asistencial.

En relación con esto el Ministerio de la Protección Social (2008) considera que las estrategias de seguridad del paciente requieren acciones coordinadas y, sobre todo, un importante cambio cultural en las organizaciones del país, lo cual significa que la seguridad del paciente resulte en "la evaluación permanente y proactiva de los riesgos asociados con la atención en salud para diseñar e implantar de manera constante las barreras de seguridad necesarias".

Para hacer frente a los EA y llevar a cabo las medidas precisas para reducirlos o evitarlos, entonces, es necesaria una cultura de seguridad dentro de las instituciones. Así, desde la concepción de Franco ${ }^{5}$, quien plantea que la cultura es un conjunto de "Creencias, actitudes y comportamientos que caracterizan las acciones de un grupo de personas dentro de una organización", la cultura de la seguridad en una organización de salud significa que cada persona del equipo de salud tenga conciencia de que la seguridad es prioritaria, y que esta se vea plasmada en todas las prácticas de los servicios de salud que presta. En consecuencia, la cultura de

5 FRANCO. Fundamentos de seguridad al paciente para disminuir errores médicos. Cali: Universidad del Valle, 2006. p. 58. 
la seguridad conlleva un proceso de educación continuada para el personal del sector, que debe estar en conexión directa con el trabajo en equipo y con la comunicación efectiva en las organizaciones. Solo de este modo la cultura de la seguridad del paciente se convierte en un proceso que les permite a las instituciones sanitarias aprender y rediseñar los procesos con el fin de que los errores no se vuelvan a cometer.

En este sentido, la cultura de la seguridad en una institución se expresa en las políticas que plantea acerca de la seguridad en la atención y en la forma en que estas se llevan o no a cabo. El Sistema de Garantía de la Calidad, entonces, se configura como un apoyo en relación con la cultura de seguridad del paciente ya que aborda el quehacer organizacional, creando en las entidades la necesidad de auto gestionarse, trabajar coordinadamente, y tener claramente definidos y organizados los objetivos con los cuales pretende darle cumplimiento a sus competencias legales y constitucionales, bajo una óptica de la calidad y de ética institucional.

Este Sistema requiere que el proceso de atención en salud tenga en cuenta las siguientes consideraciones: informar y reportar la ocurrencia de un evento adverso (EA); establecer los correctivos necesarios y hacer el seguimiento; evitar que se presenten, y si ello ocurre identificar sus causas, y medir el impacto al usuario, a la familia, a la comunidad, al personal de salud y a la propia institución. De la misma manera, para garantizar prácticas seguras en el proceso de atención al cliente asistencial y ofrecer el servicio de salud orientado al logro de altos estándares de calidad, es necesario orientar a la institución de salud en el diseño, desarrollo, implementación, seguimiento y mejoramiento de un programa que fortalezca la gestión relacionada con la identificación, investigación, notificación del riesgo y la seguridad de los pacientes.
Otro de los aspectos fundamentales para poder construir e implementar políticas de seguridad para el paciente, es el de la condición humana del personal de salud, pues el propio entorno bio-psicosocial de cada ser humano afecta la interacción personal en cuanto a comportamientos, habilidades, concentración, su forma de pensamiento y su respuesta a los procesos patológicos. Sumado a esto, en el ámbito de la atención en salud, quien interactúa no solo debe estar atento a su labor y a las relaciones personales que establece con una 0 varias personas a la vez, sino también, al funcionamiento adecuado de los demás agentes involucrados (tecnología y equipos), procesos que al ser simultáneos resultan particularmente difíciles de sostener por largo tiempo, favoreciendo la aparición del error.

Además, en relación con el reporte como práctica necesaria en una cultura de seguridad del paciente, existen dos factores dentro de la condición humana que afectan su consolidación: uno tiene que ver con las diferentes profesiones que se agrupan en el sector salud, ya que se encuentra que varios estudios, alrededor del mundo, demuestran que los médicos son más renuentes que otros profesionales de la salud a reportar eventos e incidentes; el otro factor está relacionado con la cultura institucional que tradicionalmente es punitiva frente a los errores asumidos por el personal, ya que los EA generan demandas y quejas en las instituciones de salud, por lo cual se tiende a no informarlos, lo que evita su registro y análisis.

En este sentido, Franco (2005) es ilustrativo al afirmar que:

La cultura laboral que históricamente ha rondado las instituciones de salud ha sido la de la punición, es decir, cuando sucede un error el culpable es castigado. Es claro ahora en el ámbito de la salud que la cultura punitiva genera más perjuicio que beneficio. Por otro lado, teniendo en cuenta que siempre van a existir los errores, la acción más lógica y necesaria para la organización se debe dirigir 
hacia la creación de una cultura institucional educativa y no punitiva que tenga su punto de partida en el informe y análisis de los errores y eventos adversos 6 .

De esta manera, se observa que la percepción, es decir, las creencias y actitudes, y el conocimiento del personal médico son relevantes en la creación o fortalecimiento de una cultura positiva sobre seguridad del paciente en las instituciones de salud, ya que afectan directamente el reporte de los EA, el cual es uno de los procesos esenciales para la prevención de estos, ya que conlleva la generación de aprendizaje de los errores de forma proactiva para reestructurar los procesos, y disminuye las posibilidades de que estos errores se vuelvan a producir.

\section{METODOLOGÍA}

Este apartado presenta en primer lugar el resultado de la revisión bibliográfica, para posteriormente exponer lo correspondiente al trabajo de campo. En relación con los artículos revisados, basados en trabajos de campo en el contexto latinoamericano, se encuentra que la bibliografía frente a este tema es escasa (cinco investigaciones) y que el estudio IBEAS, proyecto desarrollado por Colombia en conjunto con México, Costa Rica, Perú y Argentina, con el patrocinio de la Alianza Mundial por la Seguridad del Paciente (OMS Ginebra) y la Organización Panamericana de la Salud y el Soporte Técnico del Ministerio de Sanidad y Consumo (España), es la única investigación al respecto que aporta datos comparativos entre países de esta región.

El estudio IBEAS pretende determinar la prevalencia de EA y caracterizar los efectos adversos relacionados con la asistencia sanitaria, distinguiendo los evitables de los que no lo son. Este estudio abarca 11.379

6 FRANCO, A. La seguridad clínica de los pacientes: entendiendo el problema. En: Revista Colombia Médica, 2005, vol. 36, p. 130-133. pacientes de 58 hospitales, de los cinco países mencionados, entre los años 2006 y 2009. La investigación arrojó que la acreditación es definitiva en términos de la cultura de seguridad en el paciente, y que las instituciones participantes de Colombia tienen mayor capacidad de detección de los eventos adversos que las de los otros países.

Por otro lado, aunque IBEAS es un estudio regional, en cuanto a los otros estudios es importante señalar que dos, el de Esandi et ál. (2004) en Argentina, y el de Gaitán et ál. (2008) en Colombia, se consideran como las investigaciones inaugurales en la materia en cada uno de los países de origen, frente a lo cual se observa que en nuestro país se consolidó el interés de manera más tardía que en el país austral. Vale la pena llamar la atención sobre el hecho de que, además, estos dos estudios basaron sus encuestas en instrumentos extranjeros: mientras que Esandi et ál. (2004) adaptaron una herramienta estadounidense, realizada por Sexton y Helmreich de la Universidad de Texas, el de Gaitán et ál. (2008) tuvo como origen el estudio canadiense de Baker.

Así, ya respecto a nuestro país, se consigue observar que las metodologías y los enfoques en la cultura de la seguridad del paciente de las tres investigaciones revisadas son disímiles tanto en sus objetivos como en el tipo de población a abordar y el origen de las instituciones participantes. Aunque todas tienen como instrumento la encuesta o entrevista, Gaitán et ál. (2006) la aplican a una población de pacientes, Delgado Gallego (2007) lo hace con una población mixta (usuarios y personal de la salud) y la combina con grupos focales, mientras que Barbosa et ál. (2008) se centran en el personal médico.

Además, la investigación de Gaitán et ál. (2006) se ajusta en la incidencia y evitabilidad de los AE, mientras que las dos restantes le dan mayor trascendencia 
al aspecto de la cultura de seguridad y los EA en las entidades de salud, lo cual permite que sus resultados presenten mayor énfasis en torno a lo que afecta en sí las prácticas médicas definidas por el propio personal de la salud. Sin embargo, cabe agregar que se ubican en contextos sociales y económicos diferentes, pues mientras Delgado Gallego (2007) realiza su estudio en dos municipios del Valle, Barbosa et ál. (2008) lo hacen en Bogotá.

En relación con los resultados compartidos por ambos estudios, vale la pena mencionar dos de ellos. El primero tiene que ver con el subregistro que se presenta todavía con el reporte de los EA, pues se encuentra que este se ve fuertemente afectado por el temor que el personal de salud tiene frente a las represalias de la empresa o las legales. En segunda instancia, en ambas investigaciones se observa que las actitudes del personal de la salud frente a la seguridad del paciente son ambivalentes, ya que aunque existe favorabilidad hacia el reporte y aprendizaje del error, existen elementos situacionales que determinan cierta resistencia. Esto significa que, a pesar de que existen procedimientos y sistemas para manejar la seguridad del paciente en las instituciones, no siempre son suficientemente eficaces, ya que en ocasiones no se aplican ya sea por falta de conciencia, descuido o por exceso de trabajo.

\section{Marco normativo colombiano}

Puede considerarse que el primer antecedente legal, en términos de seguridad del paciente, es la Ley 10 de 1990, por la cual se reorganiza el Sistema Nacional de Salud, que en sus artículos 1 y 20 establece la fijación de normas de calidad para servicios de salud y los mecanismos para controlar y vigilar su cumplimiento. En complemento a esta, la Constitución Política de 1991, en sus artículos 48 y 49 expresa que "la seguridad social es un servicio público de carácter obligatorio que se prestará bajo la dirección, coordinación y control del Estado, en sujeción a los principios de eficiencia, universalidad y solidaridad, en los términos que establezca la Ley". Así, esta define al Estado como responsable de organizar, dirigir y reglamentar la seguridad social y de establecer las políticas para la prestación de servicios de salud por entidades privadas, y ejercer su vigilancia y control.

De esta manera, aparece la Ley 100 de 1993, por la cual se crea el Sistema de Seguridad Social Integral que, en su numeral 9, artículo 153 dispone que el Sistema General de Seguridad Social en Salud establecerá mecanismos de control a los servicios para garantizar a los usuarios la calidad en la atención y que esta sea oportuna, integral, continua y de acuerdo con estándares aceptados en procedimientos y prácticas profesionales. También, en el artículo 199 de esta Ley, se define que el Ministerio de Salud solicitará la información que estime necesaria con el objeto de establecer sistemas homogéneos de registro y análisis que permitan periódicamente la evaluación de la calidad del servicio y la satisfacción del usuario.

Es así como, desde 1994, en Colombia se desarrolló un sistema específico de calidad para el sector salud, cuya filosofía y orientación práctica tiene como eje la seguridad de los usuarios del Sistema. Para ello, en el Decreto 2174 de 1996, por el cual se organiza el Sistema Obligatorio de Garantía de Calidad del Sistema General de Seguridad Social en Salud, se incluye la definición de seguridad y la perspectiva de detección y control de los riesgos como eje central de la política de la calidad.

Posteriormente, el Decreto 2309 de 2002, por el cual se define el Sistema Obligatorio de Garantía de Calidad de la Atención de Salud del Sistema General de Seguridad Social en Salud, viene a reforzar este concepto al mencionar, en el artículo 7, sus cuatro componentes básicos: el sistema único de habilitación, 
la auditoría para el mejoramiento de la calidad de la atención en salud, el sistema único de acreditación y el sistema de información para la calidad. Además, en el artículo 38 del mismo Decreto, se hace énfasis en las acciones que deben emprender las IPS, especialmente, las acciones de tipo coyuntural, en términos de que estas "deben alertar, informar y analizar la ocurrencia de eventos adversos durante los procesos de atención de salud y facilitar la aplicación de intervenciones orientadas a la solución inmediata de los problemas detectados, y la prevención de su recurrencia" (Ministerio de Salud, 2002).

De igual forma, las sucesivas resoluciones reglamentarias del Decreto 1011 de 2006, en el cual se establece el Sistema Obligatorio de Garantía de Calidad de la Atención de Salud del Sistema General de Seguridad Social en Salud, y la reciente Resolución 1043 de 2006, por la cual se establecen las condiciones que deben cumplir los prestadores de servicios de salud para habilitar sus servicios e implementar el componente de auditoría para el mejoramiento de la calidad, se orientan a determinar las condiciones mínimas, sin las cuales prestar el servicio de salud puede considerarse inseguro o riesgoso.

A la vez, el programa de Auditoría para el Mejoramiento de la Calidad tiene como una de sus prioridades enfocar sus esfuerzos de mejoramiento en la seguridad, en la detección de riesgos y fallas de la atención; mientras que en la Resolución 1446 de 2006, en la cual se define el Sistema de Información para la Calidad y se adoptan los indicadores de monitoría del Sistema Obligatorio de Garantía de Calidad de la Atención en Salud, el sistema de información adquiere mecanismos para conocer los principales eventos y sus causas, y para facilitar la elaboración de diversas herramientas de promoción de la seguridad. Asimismo, esta define los llamados Eventos Adversos Trazadores, los cuales están destinados a orientar a las instituciones sobre aquellos eventos a los cuales deben prestar mayor atención, con el fin de prevenirlos. De esta forma, todas estas medidas constituyen avances importantes y se convierten en un recurso inicial, de mucho valor, puesto que, además, cobijan también al sector asegurador.

En esta línea, el logro más importante se dio a partir de la inclusión, en el Sistema Obligatorio de Garantía de Calidad, de la noción de eventos adversos y del desarrollo del sistema de acreditación. Este le da especial relevancia a la seguridad del paciente, ya que define, como requisito de entrada, el desarrollo de un sistema de reporte de los eventos adversos. De tal forma, las instituciones de salud que quieran demostrar el cumplimiento de niveles superiores de calidad, se ven obligadas a demostrar que han desarrollado una cultura de prevención y control de los eventos adversos, la cual incluye, entre otros elementos, políticas específicas para estimular el reporte, definición de mecanismos no punitivos para su intervención, alarmas y respuestas inmediatas ante su ocurrencia y, sobre todo, la definición de mecanismos para prevenirlos y evitar su repetición en caso de que se presenten.

Finalmente, todas las condiciones legales llevan a considerar los eventos adversos en su justa medida, tal como el Ministerio de la Protección Social lo expresa:

La política de seguridad del paciente, no fomenta la impunidad cuando la acción insegura se deba a negligencia 0 impericia 0 haya intención de hacer daño, y respeta por lo tanto el marco legal del país. Pero de igual manera procura proteger al profesional que de manera ética y responsable, analiza las fallas de la atención y los eventos adversos para desarrollar barreras de seguridad que protejan al paciente?

7 MINISTERIO DE LA PROTECCIÓN SOCIAL, 2008, p. 12. 


\section{METODOLOGÍA}

Esta investigación es un estudio de tipo descriptivo realizado en una IPS (Institución Prestadora de Servicios de Salud) de la ciudad de Medellín, cuyo fin es el de indagar el tema de la percepción y el conocimiento del personal médico y paramédico alrededor del tema del reporte de los eventos adversos y la cultura de seguridad del paciente. En este sentido, trata de analizar el conocimiento y la información que tiene el personal frente al tema, compromiso, cultura del reporte, cultura de seguridad, prácticas seguras y comunicación, entre otras, a partir de la aplicación de una encuesta.

En este sentido, se obtuvo una muestra de número aleatorio sistemático, se calculó la muestra con un nivel de confianza del $95 \%$, un valor estimado del $50 \%$ y un error aceptable del 5\%. El tamaño de la muestra se estimó para cada uno de los grupos considerados, y utilizando una tabla de números aleatorios se identificaron las unidades muestrales desde la lista de profesionales de la institución y por cada estrato en la población susceptible de encuestar.

Así, la muestra de la población, por cada estrato, fue de 173 personas entre médicos, representados por médicos generales, rurales y especialistas; y personal paramédico, representado por enfermeras profesionales, auxiliares de enfermería, instrumentadoras quirúrgicas, camilleros y una psicóloga. En este sentido, se encuentra que las áreas con mayor representación fueron la de especialidades quirúrgicas y la clínica.

Para la recolección de datos se tomó como base el instrumento Hospital Survey on Patient Safety Culture (2004), construido por la Agency for Healthcare Research and Quality-AHRQ, de Estados Unidos, el cual, previa autorización, fue adaptado al medio y se le hicieron ajustes y se introdujeron nuevas preguntas.
Así, la recolección de datos realizada durante los meses de abril a julio de 2010 se efectúo a través de la encuesta adaptada, la cual indagó sobre el reporte de los eventos adversos y de la seguridad del paciente mediante 95 preguntas, de las cuales 81 fueron diligenciadas a través del método Escala Likert (tabla 2), por medio del cual se les solicita a los encuestados que expresen su acuerdo o desacuerdo frente a cada pregunta. A estas respuestas se les asignó un puntaje a fin de clasificarlas según reflejan actitudes positivas, negativas 0 indecisas.

La encuesta está dividida en siete niveles o componentes a indagar: Nivel A (normatividad: adherencia a normas y guías), Nivel B (factores humanos: actitudes, prácticas), Nivel C (supervisión), Nivel D (influencias organizacionales), Nivel E (legislación), Nivel F (antecedentes), y Nivel G (cultura de seguridad).

Posterior al diseño del instrumento, se procedió a realizar una prueba piloto con un grupo de 10 personas que trabajan en la institución y que se desempeñan en las áreas de cirugía, hospitalización, urgencias y administrativa. El tiempo promedio para responder la encuesta fue de 15 minutos por persona. A partir de los resultados obtenidos en esta prueba se realizó una revisión y análisis del instrumento (encuesta), de manera que la información obtenida de esta prueba piloto fue utilizada para modificar la encuesta y para desarrollar el instrumento final de la investigación.

La IPS elegida para la realización del trabajo de campo es una institución prestadora de servicios de salud de alta complejidad, habilitada por la Dirección Seccional de Salud de Antioquia. Atiende pacientes del régimen contributivo, régimen subsidiado, medicina prepagada y pacientes particulares, con capacidad para ofrecer a la comunidad una amplia gama de servicios y de especialidades médicas. Su infraestructura dispone de 5 pisos de hospitalización con 181 camas; 9 quirófanos 
para cirugía general y de las diferentes especialidades; 2 quirófanos para cirugía ginecoobstétrica; 1 sala de partos y 1 sala de trabajo de parto, con capacidad de 6 cubículos para atención a pacientes obstétricas. Además, cuenta con 2 unidades de cuidados intensivos para adultos y neonatal, respectivamente, junto con el servicio de urgencias. También, ofrece servicios de apoyo como son el de laboratorio clínico y banco de sangre, rayos X e imagenología, terapia respiratoria, terapia física y rehabilitación, oficina de apoyo y atención al usuario, entre otros.

El personal médico y paramédico que trabaja en la institución está conformado por 386 personas (tabla 1), entre socios y empleados vinculados en su gran mayoría por contrato a término indefinido, mientras que unos cuantos lo están por cooperativa.

Tabla 1. Descripción de la población en estudio:

Análisis de la percepción y el conocimiento del personal médico y paramédico de una IPS de alta complejidad en la ciudad de Medellín, respecto al reporte de los eventos adversos y la seguridad del paciente. Años 2009-2010.

\begin{tabular}{cccc}
\hline Estrato & Cablación & $\begin{array}{c}\text { Características } \\
\text { Participación }\end{array}$ & Muestra \\
\hline Auxiliares enfermería & 223 & $55,33 \%$ & 91 \\
Médicos socios & 118 & $29,28 \%$ & 48 \\
\hline Enfermeras & 44 & $10,92 \%$ & 18 \\
\hline Otros (instrumentadoras) & 8 & $1,99 \%$ & 8 \\
Camilleros & 5 & $1,24 \%$ & 5 \\
Rurales & 3 & $0,74 \%$ & 3 \\
\hline Auxiliares Comité Infecciones & 2 & $0,50 \%$ & 2 \\
\hline
\end{tabular}

Fuente: autora.

Así, se obtuvo una muestra en la población, por cada estrato, de 173 personas entre médicos, representados por médicos generales, rurales y especialistas; y personal paramédico, representado por enfermeras profesionales, auxiliares de enfermería, instrumentadoras quirúrgicas, camilleros y una psicóloga.

\section{RESULTADOS}

Con el fin de presentar de manera organizada los resultados, es importante iniciar con el análisis del Nivel F de la encuesta, en el que se indaga acerca de los antecedentes del personal (profesión, tiempo de permanencia en la institución y de ejercicio de la profesión, y tipo de vinculación laboral), para posteriormente tratar cada uno de los otros seis niveles o componentes de manera más específica.

Así, se observa que la relación entre el tiempo que los encuestados llevan trabajando en la institución y el tiempo que llevan de ejercicio profesional (Tabla 2), se encontró que la mayor parte de las personas encuestadas es muy joven laboralmente, ya que 85 de los 173 encuestados han estado trabajando menos de 10 años y en ese mismo periodo han laborado en esta institución; de igual forma sucede con las personas que llevan laborando entre 11 y 20 años. Estos datos dan indicios tanto de la baja rotación del personal en esta institución, como del hecho de que la mayor parte de los empleados iniciaron su vida profesional en esta. 
Tabla 2. Tiempo trabajado en la institución versus tiempo trabajado en la actual profesión del personal médico y paramédico encuestado de la IPS de alta complejidad en la ciudad de Medellín. Años 2009-2010.

\begin{tabular}{ccccccc}
\hline & \multicolumn{6}{c}{ Tiempo que lleva trabajando en su profesión } \\
\hline Años en la institución & $1-10$ & $11-20$ & $21-30$ & $31-40$ & $41-50$ & Total general \\
$1-10$ & 85 & 11 & 2 & & & 98 \\
$11-20$ & 1 & 36 & 5 & 1 & 43 \\
$21-30$ & & 1 & 15 & 3 & & 19 \\
$31-40$ & & & & 2 & 1 & 3 \\
Sin responder & & & & & & 10 \\
\hline Total & 86 & 48 & 22 & 6 & 1 & 173 \\
\hline
\end{tabular}

Fuente: autora.

Esto se convierte en una ventaja para la IPS, ya que significa que las personas conocen bien la institución, lo cual plantea que se puedan alcanzar mejores resultados en cuanto al tema de cultura de seguridad.

\section{Nivel A: normatividad, adherencia a normas y guías}

A continuación se presenta un análisis general de los resultados obtenidos en este nivel, en el cual se hicieron 15 preguntas cuyo objetivo fue explorar si el personal encuestado conoce y practica las normas, guías y protocolos de atención, además si conoce y aplica las prácticas seguras en las labores diarias de trabajo para el desarrollo de una cultura institucional en seguridad del paciente, como punto de partida para comenzar el camino hacia la seguridad.

Entre las fortalezas en este nivel, se encontró que la mayoría de los empleados encuestados reconocen que los estudiantes deben estar previamente entrenados en la práctica de cualquier procedimiento y que, además, tienen una percepción positiva acerca de la preparación de los empleados para hacer las tareas encomendadas; es decir, que existe conciencia acerca de la necesidad de un entrenamiento adecuado como condición para evitar la ocurrencia de eventos adversos.
En una tercera parte de los interrogantes planteados en este nivel se encontró que el promedio es inferior al punto de corte (que se asume como 4.0), lo cual significa que aparece una debilidad importante, representada en el hecho de que los médicos aceptan no conocer ni manejar las guías de atención clínica (preguntas A11 y A12), de forma contraria a lo que ellos manifiestan que sucede con el personal de enfermería (preguntas A7 y A8).

Así, los médicos afirman que el 77,09\% (es decir, la suma de los porcentajes de médicos que califican $4 \mathrm{y}$ 5) de las enfermeras sí conoce las guías de enfermería que existen en la institución, además que un 70,21\% (suma de los porcentajes que califican 4 y 5 ) de ellos afirma que las enfermeras de la institución se adhieren a estas guías. En relación con la seguridad del paciente, estos porcentajes son muy positivos.

En contraste con lo anterior, la percepción de las enfermeras respecto al conocimiento y manejo de las guías de atención, por parte de los médicos, no es tan positivo. Las enfermeras afirman que solo el 47,75\% de los médicos conocen las guías de atención (pregunta A11) y tienen una percepción muy similar frente al manejo de las guías por parte de los médicos, ya que se reconoce en solo un $43,75 \%$. Además, las enfermeras 
encuestadas manifiestan que el 19,82\% de los médicos no conoce las guías de atención y que el 11,66\% de ellos no manejan las guías de atención. Una tercera parte de las enfermeras encuestadas se encuentran indecisas sobre estos interrogantes.

Sin embargo, en términos generales para el 78,24\% de los encuestados el personal de salud conoce los protocolos de manejo establecidos en la institución (pregunta A6), lo cual representa un porcentaje muy significativo.

En cuanto a este punto se realizó un cruce entre el oficio y el conocimiento de las normas de seguridad establecidas, con el objetivo de conocer la percepción según el oficio.

Así, se encontró que las enfermeras profesionales y los médicos especialistas presentaron los valores promedio más bajos (3,6 y 3,8, respectivamente), mientras que para otros oficios como instrumentadoras, camilleros y enfermeras auxiliares la calificación es superior a 4. Estos promedios llaman la atención, debido a la responsabilidad que tienen las personas de estas profesiones dentro de la institución de salud. Esto puede tener una explicación en términos de que el sentido de autocrítica es mayor en las personas que ocupan cargos de mayor jerarquía.

En otro punto importante, se observa que más de la mitad de los empleados encuestados $(56,73 \%)$ reconocen informar del incumplimiento de las normas por parte de otros empleados (pregunta A9), lo cual se ratifica con el resultado de la pregunta A2 (62,21\%), en la que se indaga acerca de si las acciones inseguras pasan desapercibidas. Sin embargo, se encuentra que el 69,01\% del personal encuestado reconoce que las normas de seguridad establecidas en los procesos de atención se aplican (pregunta A5).
Otro resultado importante tiene que ver con la percepción de los encuestados sobre si se cuestiona a un miembro del equipo cuando toma decisiones por cuenta propia, que puede conllevar un riesgo para el paciente (pregunta A10), pues se encontró que el $75,29 \%$ de los encuestados afirman que esto se hace. Es de resaltar que lo percibido por el personal en esta pregunta es de gran importancia respecto al tema de la cultura de seguridad, ya que aunque tomar decisiones y conductas propias, que pueden ocasionar riesgos para los pacientes, en ciertos casos es necesario, estas no deben convertirse en conductas repetitivas ya que pueden ocasionar un riesgo en la vida de un paciente e incidentes o eventos adversos. El resto del personal en un 17,06\% está indeciso y el 7,64\% afirma que no lo hace.

\section{Nivel B: factores humanos y actitudes}

El Nivel B abarca los factores humanos y actitudes, de manera que se busca conocer qué tanto puede incidir el entorno bio-psico-social en la conducta del individuo, en su conocimiento y en la práctica profesional. Para este nivel, el 60\% de los promedios obtenidos se ubicaron por debajo del punto de corte $(4,0)$, con un promedio general de 3,7 .

Entre las fortalezas en este nivel se encontró que la mayoría de los empleados encuestados afirman que el personal identifica y resuelve rápidamente situaciones de emergencia que se presentan en el trabajo (preguntas B1, B2, B3). Factor importante en el momento de atención de un paciente, ya que se percibe que el equipo de salud está en una condición llamada "alerta situacional", es decir, que está atento en todo momento para actuar frente al mínimo cambio que pueda presentar el paciente.

Otra fortaleza manifestada tiene que ver con que los encuestados afirman que el tener un trato digno, respetuoso y amable hacia los pacientes atendidos hace 
parte importante del componente humano del personal de salud (preguntas B23, B24 y B25). En relación con esto, se detectó que la mayoría de los encuestados (91,33\%) aceptan las correcciones y sugerencias dadas cuando se comete un error, lo cual implica que existe una cultura del aprendizaje (pregunta B18).

Se encontraron interrogantes que aparecen por debajo del promedio general $(3,7)$ en este nivel, que son importantes de analizar.

Así, frente a la pregunta sobre la posibilidad de desligarse de las emociones y sentimientos durante el proceso de atención (B21), más de la mitad del personal encuestado $(59,17 \%)$ manifiesta lograrlo mientras que un 20,32\% refiere no lograrlo.

Por otra parte, frente a la posibilidad de lograr sus tareas sin afanes (pregunta B10), el 46,47\% del personal refiere que se toma el tiempo necesario para realizarlas, mientras que el 28,82\% refiere no lograrlo. De cierta forma, estos porcentajes marcan una tendencia no favorable, ya que en salud no tomar el tiempo necesario para realizar los procedimientos sin afanes posibilita que las personas cometan errores, lo cual facilita que sucedan incidentes y eventos adversos indeseables.

Uno de los puntos clave en el reporte de EA hace referencia a la percepción del personal sobre si sus errores son usados en su contra (pregunta B5), lo cual es sentido así por el 36,41\% del personal encuestado, mientras que el 30,06\% no considera que esto sea así. Llama la atención que un 33,53\% de los encuestados están indecisos en la respuesta (calificación de 3 ).

Las preguntas B19, B6, B16, B4, B20, B17 y B14 presentan un promedio inferior muy cercano al punto de corte de 4, lo cual hace relevante mencionarlos. Frente a la opinión que el personal tiene en relación con la disponibilidad adecuada de los equipos, se encuentra que el 68,82\% afirma que se cuenta con todos los equipos necesarios para la atención y cuidado del paciente (pregunta B19), y para el 75\% estos equipos están en buen estado (pregunta B20).

En cuanto al entrenamiento del personal temporal (pregunta B6) el 68,82\% de los encuestados manifies$\tan$ que este se realiza. Es de recordar que la gran mayoría del personal en la institución es estable.

Uno de los factores primordiales en la seguridad al paciente es la comunicación que exista entre el equipo de salud encargado de la atención del paciente, ya que cada miembro del equipo maneja su propia información, que si se comparte puede mejorar un proceso y minimizar los riesgos de errores. Al indagar sobre la precisión en la comunicación del equipo de trabajo se encontró que un 66,66\% afirma entablar una buena comunicación, mientras que un $17,54 \%$ señala no estar de acuerdo con que existe una buena comunicación (pregunta B9).

En relación con esto, el 69,82\% afirma que los jefes se preocupan cuando ocurren problemas con respecto a la seguridad del paciente (pregunta B16), lo cual es muy positivo para la institución y para el personal que labora en esta. Además, el 77,78\% de los encuestados afirma que su jefe acepta las sugerencias del personal para implementar mejoras en cuanto a la seguridad de los pacientes (pregunta B14). Estas percepciones dan más indicios sobre la existencia de una cultura de aprendizaje en la institución. Sumado a esto, para el 70,35\% de los encuestados, cuando se necesita terminar una gran cantidad de trabajo, se hace en equipo (Pregunta B4).

En cuanto a si el personal de salud del servicio se distrae fácilmente con otras cosas como la música, crucigramas, libros de lectura, entre otros (pregunta B17), la mayoría de las personas encuestadas (73,26\%) están de acuerdo en que realizan su trabajo de manera concentrada, mientras que un 12,79\% de las personas encuestadas se distraen fácilmente. 
Finalmente, un aspecto decisivo en la cultura de la seguridad tiene que ver con la preparación en el momento de atender un paciente en estado crítico, frente a lo cual el 66,27\%, de los encuestados afirman tener pertinencia en el tema, mientras un $15,98 \%$ afirman estar indecisos y un 17,75\% afirman estar en desacuerdo y muy en desacuerdo (pregunta B13). Este punto es clave ya que las salas de cuidados intensivos junto con las salas de cirugía, servicios de urgencias, salas de exámenes y procedimientos invasivos son catalogados como servicios de alto riesgo. Los adelantos tecnológicos y científicos hacen de este aspecto un desafío continuo para las instituciones y las personas del equipo de salud que laboran en estas, en cuanto a la seguridad del paciente.

\section{Nivel C: supervisión}

El nivel supervisión es importante para tener en cuenta en la institución cuando se vincula el personal que tiene poca experiencia o es muy nuevo en su profesión, pues el seguimiento constante, los procesos de inducción y supervisión, la información de riesgos y la auditoría, entre otros, son fundamentales en la cultura de seguridad. Este nivel tiene como objetivo identificar si el personal de la institución acepta la supervisión como aspecto importante y obligatorio en el tema de seguridad del paciente. El promedio general obtenido en este nivel corresponde a 3,6, de la misma forma que en los niveles anteriores se tomó 4 como punto de corte.

Es relevante comenzar con los procesos de inducción en la institución para el personal que ingresa a ella (pregunta C5), pues el promedio obtenido fue de 4,3, lo cual es un paso importante para que todo el personal conozca y trabaje bajo los mismos parámetros evitando que se cometan errores y mejore la calidad de atención en salud. Además, se encuentra que cuando se presenta una situación de riesgo esta es reportada por cualquier persona del equipo (pregunta C7), ya que el promedio obtenido según los encuestados es de 4,2. De otro lado, los encuestados perciben que la institución acostumbra a hacer auditoría permanente (pregunta C6), con el fin de mejorar cada día los procesos, dando una calificación promedio de 4,1. Todos estos aspectos minimizan riesgos, ya que si se realiza sistemáticamente una gestión de seguridad, de procesos y actividades en la institución, se corrigen a tiempo las desviaciones de los estándares en los que se basa el programa de seguridad del paciente de la institución.

Aunque aparezca por debajo del punto de corte de este nivel, la pregunta C2 ratifica lo observado en los datos anteriores, ya que se encuentra que el 27,48\% de los encuestados afirma estar en desacuerdo y muy en desacuerdo con que el personal de salud debe ser supervisado mientras realiza su trabajo, mientras que el 46,79\% le parece correcto que supervisen su trabajo; es decir, casi el 50\% de los encuestados se siente seguro con su trabajo y no le molesta ser supervisado.

A continuación se analizan los otros puntos que están por debajo del punto de corte de este nivel, ya que son relevantes. Así, es muy importante y de obligatorio cumplimiento para cualquier entidad de salud tener registros en la historia clínica, de una forma precisa y clara, de las órdenes impartidas por los médicos tratantes, no solo por la continuidad del tratamiento sino por aspectos tan importantes como la información, comunicación y seguridad, además de tener los soportes necesarios en caso de cualquier situación que se presente con posterioridad. Frente a esto, más de la mitad de los encuestados $(56,63 \%)$ afirma que las órdenes son impartidas en forma escrita (pregunta C3), mientras que un 28,11\% afirma lo contrario.

En algunas oportunidades, las personas tienen la costumbre de revisar o hacer algún tipo de chequeo así otra persona lo haya hecho, lo cual es una buena práctica que debería instaurarse como norma de seguridad 
en las instituciones de salud. Frente a esto, el 59,28\% de los encuestados afirma que en su servicio algún miembro del equipo revisa nuevamente o practica algún tipo de chequeo de las condiciones inherentes a la actividad que se realizó (pregunta C1).

Por otro lado, el 59,65\% de los encuestados manifiesta que en el equipo de salud del cual hacen parte, las responsabilidades y tareas son repartidas equitativamente (pregunta C4).

\section{Nivel D: influencias organizacionales}

Este nivel resalta algunos elementos que están estrechamente relacionados con la cultura de la institución, tales como las políicicas, misión, visión, procesos, entre otros, que hacen que el personal que labora en la institución ejecute las actividades asignadas de una manera similar. El 80\% de las preguntas evaluadas en este nivel presentan una calificación inferior al punto de corte de 4 , con un promedio general de 3,6.

Entre las fortalezas de este nivel, se observa que los encuestados afirman que la organización desarrolla procesos que garantizan a los usuarios una atención segura (pregunta D5), seguida por la percepción acerca de que la institución ha motivado al personal de salud para que se preocupe por el tema de cultura de seguridad y seguridad del paciente (pregunta D1).

Las siguientes preguntas obtuvieron calificaciones por debajo del punto de corte:

Frente a la coordinación que debe haber entre los diferentes servicios en la institución (pregunta D2), el $45,29 \%$ de los encuestados manifiesta observarla mientras que para el 27,06\% esta no es muy clara, con un porcentaje similar $(27,65 \%)$ que expresa su inexistencia. En contraste, para el 54,49\% del personal encuestado existe continuidad en los tratamientos, cuando los pacientes son trasladados de un servicio a otro (pregunta D3) y para el 64,16\% de las personas encuestadas los cambios de turnos no generan problemas para los pacientes en la institución (pregunta D11). Estos datos explican el alto porcentaje ( $74,85 \%)$ frente a la percepción de que los servidores de la institución trabajan en conjunto para propiciar el mejor cuidado a los pacientes (pregunta D10). Como se observa, aunque hay datos dispares que revelan que existen algunas fallas en cuanto a la coordinación, en general se tiene un nivel de percepción aceptable a pesar de las debilidades.

De forma similar, el 74,85\% de los encuestados afirman que la dirección de la institución propicia un ambiente laboral que promueve la seguridad del paciente (pregunta D12). Así, para el 57,65\% de los encuestados afirman que la dirección de la institución se muestra interesada en la seguridad del paciente después de que ocurre un incidente 0 un evento adverso, situación muy positiva y alentadora por parte de la institución de salud; mientras que un 25,89\% de los encuestados afirman que la institución solo se interesa por el paciente en el momento en que ocurre el incidente (pregunta D9).

La comunicación y el buen ambiente de trabajo contribuyen a la unidad de equipo, con un propósito común: el bienestar del paciente. En las preguntas D4, D6, D7, que hacen referencia a este tema en particular, trabajo en equipo, intercambio de la información y cooperación, entre los diferentes servicios de la institución más de la mitad de los encuestados $(53,22 \%)$ afirman que hay buena cooperación entre los servicios que requieren trabajar conjuntamente (pregunta D4); en la pregunta D7, donde se cuestiona acerca del tema del intercambio de información entre los servicios de esta institución, el 52,08\% de los encuestados afirman existe y, finalmente, para el 62,94\% de los encuestados es agradable trabajar con personal de otros servicios en esta institución (pregunta D6). 
De esta manera, para el 74,85\% de los encuestados la institución tiene procesos administrativos debidamente diseñados y que funcionan, en caso de que el personal requiera orientación con respecto al tema de seguridad del paciente, reporte de eventos adversos o se sientan motivados e interesados por la cultura de seguridad en la institución, entre otros (pregunta D15).

\section{Nivel E: legislación}

Este nivel pretende indagar en el grado de conocimiento que el personal de la institución tiene en relación con la legislación en la materia. Se encuentra de esta forma que un muy bajo porcentaje $(0,58 \%)$ de los encuestados afirma estar en desacuerdo y muy en desacuerdo en que la seguridad del paciente debe ser un tema obligatorio en las instituciones de salud, respecto a un $99,42 \%$ que afirma estar de acuerdo o muy de acuerdo en que la seguridad del paciente debe ser un tema obligatorio en las instituciones de salud. Es decir, casi la totalidad de los encuestados piensan que la seguridad del paciente debe ser un tema obligatorio en las instituciones de salud dado que la calificación promedio fue de 4,7 (pregunta E3).

En segunda instancia, la mayor parte de los encuestados piensa que el Ministerio de la Protección Social debe ser más exigente con las instituciones de salud para que cumplan con la información que se les exige (pregunta E5), seguido por estar de acuerdo con que la cultura de seguridad del paciente en los trabajadores del sector salud debe estar enfocada a mejorar la calidad de los servicios (pregunta E4). Por último, los encuestados opinan que las entidades reguladoras (Supersalud y las secretarías de salud) deben sancionar a las instituciones que no cumplen con las políticas de seguridad (pregunta E6).

El 74,69\% de los encuestados sabe qué es el Sistema Obligatorio de Garantía de Calidad de la Atención de Salud, del Sistema General de Seguridad Social en
Salud (figura 8), lo cual está en concordancia con el porcentaje de encuestados, el 77,85\% opinan que el Ministerio de Protección Social ha enfocado la atención en salud en la promoción de atención en salud segura.

\section{Nivel G: cultura de seguridad}

Este nivel pretende indagar sobre los distintos aspectos que configuran la cultura de la seguridad en la institución. Así, el 35\% de las preguntas no superan la línea de corte, y el promedio general del nivel supera la línea de corte con una calificación de 4,1.

Los encuestados están de acuerdo en que al paciente se le debe informar la finalidad del tratamiento terapéutico que se le va a suministrar (pregunta G7) ya que el promedio obtenido en esta pregunta fue de 4,7, al mismo tiempo que concuerdan en que se le debe informar de manera segura y actualizada acerca de la asistencia clínica que se le va a ofrecer (pregunta G6), ya que el punto tiene un promedio de 4,6.

La institución dispone de un sistema de comunicación y análisis de incidentes que ocurren en los procesos de atención en salud y sus posibles causas, ya que el promedio obtenido en este interrogante fue de 4,1 (pregunta G1). Así, se encontró que cuando ocurre un incidente 0 un evento adverso, el personal implicado asume la situación ya que su calificación fue de 4 (pregunta G3). En relación con esto, los encuestados están de acuerdo que al ocurrir un incidente 0 un evento adverso en el proceso asistencial se debe informar y colaborar con el proceso de aprendizaje de todo el equipo de salud del cual hace parte, dado que su promedio es de 4,2 (pregunta G2).

Además, se observa que existe confianza en los mecanismos que refuerzan la cultura de seguridad del paciente, ya que el 72,19\% de los encuestados piensan que la institución dispone de mecanismos oportunos 
y eficaces de retroalimentación, acerca de recomendaciones y prácticas seguras para la atención del paciente (pregunta G5); y se tiene un promedio alto $(4,5)$ en relación con la percepción acerca de que el reporte de incidentes, errores o eventos adversos influye en el mejoramiento de la seguridad del paciente (pregunta G8). Por esta razón, se entiende que la mayor parte de los encuestados considere que para aprender de las fallas es necesario trabajar en un ambiente educativo y no punitivo, pero a la vez de responsabilidad y no de ocultamiento, ya que la calificación obtenida es de 4,4.

En este sentido, se encontró que los encuestados afirman conocer el instrumento, que utiliza la institución, para el reporte de los incidentes y los eventos adversos que ocurren en el proceso de atención en salud, dado que su calificación fue de 4,2 (pregunta G9).

Se observa que el 78,23\% de los encuestados afirman que existen normas para actuar cuando ocurre una situación inesperada en el trabajo (pregunta G4). El 68,82\% de las personas encuestadas afirman que cuando se comete un error en el proceso de atención, por parte del personal de salud en la institución, este es aceptado (pregunta G11). De allí que la mayor parte de los encuestados (59,31\%) estén de acuerdo o muy de acuerdo con que en la institución se percibe un clima de confianza y seguridad cuando se reporta o comunica un incidente 0 evento adverso. Esta percepción se ratifica ya que tan solo un $15,7 \%$ afirman no sentirse tranquilos ante esta situación (pregunta G10).

Por otro lado, también se pudo determinar que el $74,41 \%$ de los encuestados perciben que la organización destina recursos para la seguridad del paciente (pregunta G13).

A pesar de que los encuestados consideran que la cultura de la organización influye en la percepción de los valores y creencias, y en los comportamientos de las personas que trabajan en ella, pues esta pregunta tiene un promedio de 4,2 , se encuentra que existe un porcentaje considerable de los encuestados que no comunican los eventos adversos o incidentes debido a posibles medidas disciplinarias con un 35\%, seguido por falta de tiempo y repercusión ético-legal, con un $26 \%$, repercusiones ético-legales con $13 \%$ y desconocimiento del tema un $13 \%$. Así, se observa que el mayor obstáculo para el reporte es el temor a medidas disciplinarias (figura 1).

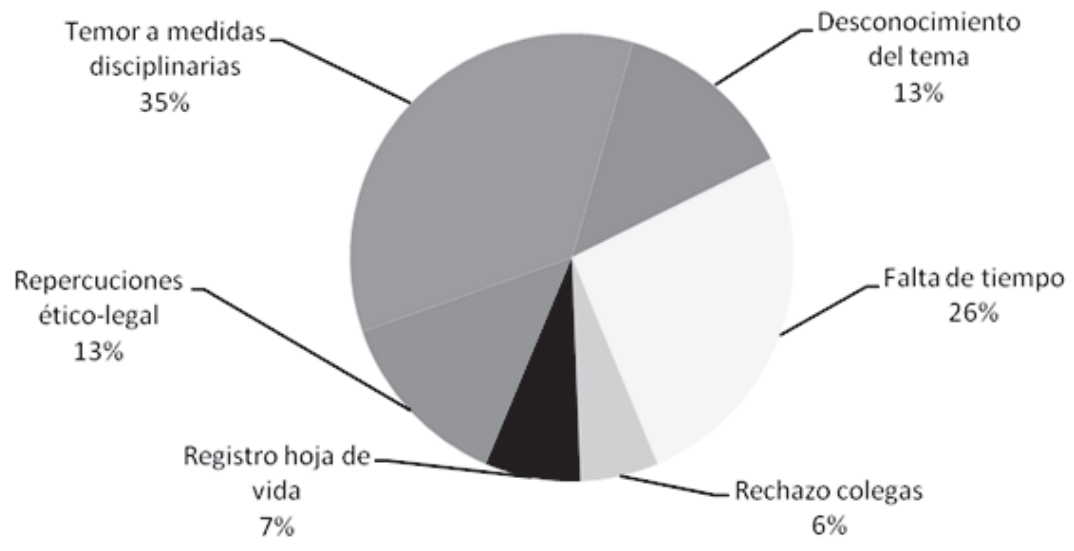

Fiǵura 1. Reporte del evento adverso o incidente por parte del personal médico y paramédico encuestado en la IPS de alta complejidad en la ciudad de Medellín, respecto al reporte de los eventos adversos y la seguridad del paciente. Años 2009-2010.

Fuente: autora. 
Aunque falte lograr mayor reporte, cerca del 70\% del personal encuestado percibe la seguridad del paciente como excelente y muy buena, un 31,21\% como aceptable y un porcentaje muy bajo $(0,58 \%)$ la considera mala o regular. Ahora bien, esta percepción varía según el área, ya que respecto a la seguridad del paciente en el servicio de urgencias, el 86,37\% de los encuestados afirman que los pacientes se sienten seguros con la atención que se brinda en el servicio, mientras que el $75 \%$ del personal encuestado de la unidad de cuidados intensivos afirman que los pacientes se sienten seguros con la atención en el servicio.

En relación con esto, se realizó un cruce entre la pregunta E3 (del nivel de legislación) en la cual se interroga al encuestado sobre si la seguridad del paciente debe ser un tema obligatorio en las instituciones de salud, con la G19 en la que se pregunta si piensa que todas las normas de seguridad deben ser obligatorias. Así se obtuvo que el 70,93\% del personal encuestado expresan que la seguridad del paciente debe ser un tema obligatorio en las instituciones de salud y piensan que todas las normas de seguridad deben ser obligatorias.
La Política de Seguridad del Paciente es un conjunto de acciones y estrategias que ofrece el Sistema Obligatorio de Garantía de Calidad para proteger al paciente de riesgos evitables que se derivan de la atención en salud. Al indagar al personal sobre su conocimiento acerca de la política de seguridad que tiene la institución, 84,38\% de los encuestados afirman conocerla, y tan solo un 15,61\% no la conocen. En este sentido, el 63,91\% de los encuestados afirman haber participado en capacitaciones acerca del tema de cultura de seguridad del paciente (pregunta G18).

\section{DISCUSIÓN}

En este punto se analiza la relación entre los niveles presentados anteriormente. Así, se encuentra que los niveles legislación y cultura de seguridad superaron el punto de corte establecido (4), mientras que los niveles de normatividad, factores humanos, supervisión e influencias organizacionales no superaron el punto de corte (figura 2).

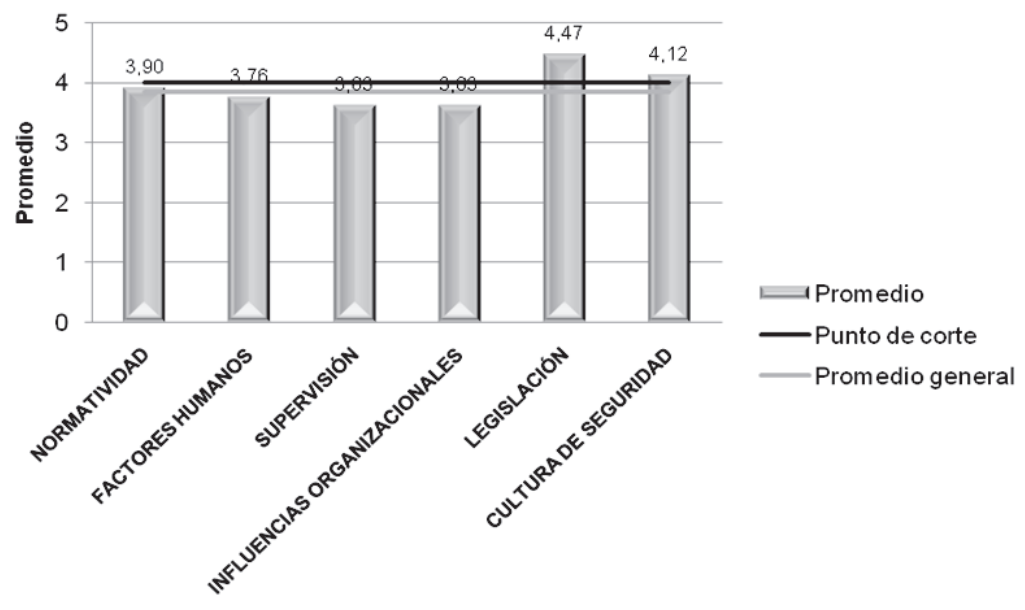

Figura 2. Niveles en el estudio de la percepción (creencias y actitudes) en una IPS de alta complejidad en la ciudad de Medellín, respecto al reporte de los eventos adversos y la seguridad del paciente. Años 2009-2010.

Fuente: autora. 
Aunque la calificación de estos niveles está ubicada muy cerca al punto de corte, es necesario establecer planes de acción en estas áreas para mejorar todos estos aspectos que son importantes y que constituyen pilares significativos para la cultura de seguridad de la institución, por lo cual se requiere de un compromiso y un deber por parte de la institución como centro seguro, por parte del personal médico y paramédico con la cultura de desplegar buenas prácticas en salud, y el compromiso del paciente y la sociedad que aunque siempre se han considerado como observadores, actualmente, es necesario vincularlos y comprometerlos por su seguridad cuando asisten a las instituciones de salud en pos de una atención.
En el análisis de actitudes, prácticas y conocimientos se encontró que cada una de estas capacidades está por debajo del punto de corte establecido (figura 3). Estos tres aspectos son pilar fundamental en todas las personas que trabajan en el sector salud, específicamente en todo lo que tiene que ver con el tema de cultura de seguridad y reporte de los eventos adversos. Así, se hace relevante revisar en cada uno de ellos cuáles son las debilidades y necesidades de cambio necesarias, para plantear soluciones que lleven a transformar e implementar cambios necesarios, para una mejor actitud en el personal.

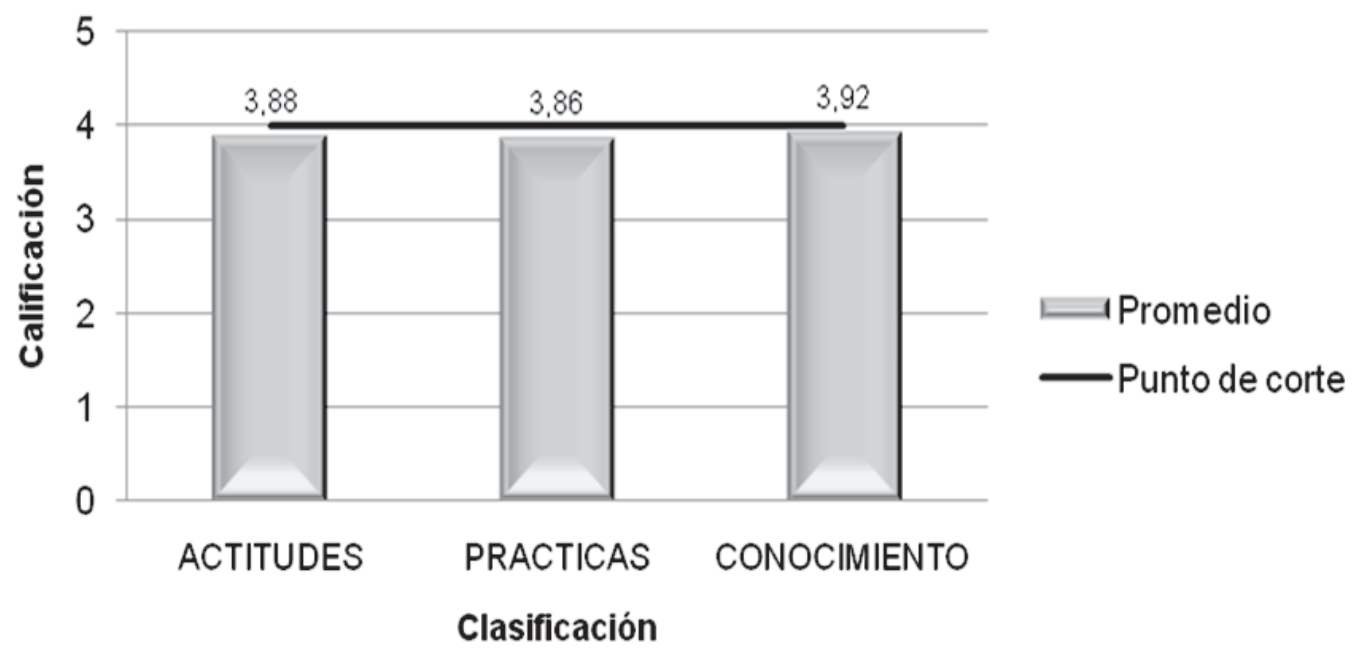

Figura 3. Análisis de actitudes, prácticas y conocimientos de una IPS de alta complejidad en la ciudad de Medellín, respecto al reporte de los eventos adversos y la seguridad del paciente. Años 2009-2010.

Fuente. autora.

\section{V.CONCLUSIONES}

La relación entre el conocimiento de los procedimientos, la normatividad y la legislación y su respectiva aplicación permite deducir hasta qué punto existe una cultura de la seguridad arraigada o si esta presenta debilidades importantes. Así, frente a la normatividad, aunque para el 78,24\% de los encuestados el personal de salud de la institución conoce los protocolos de manejo establecidos, se encuentra que las personas con niveles educativos altos (médicos especialistas, enfermeras profesionales) presentan menor grado de conocimiento de las normas de seguridad establecidas por la institución, frente a una mayor apropiación de estas por parte de otros oficios como instrumentadoras, camilleros y enfermeras auxiliares. 
En este sentido, se observa que a pesar de que la mayoría de los empleados conoce la normatividad y la legislación, y este porcentaje no es insignificante, en relación con la presencia de una cultura de seguridad, es un dato inquietante, ya que muestra que por lo menos un 20\% del personal desconocen la normatividad. Además, se destacan en esta vía las diferencias según el oficio, aspecto preocupante si se tiene en cuenta el hecho de que si bien el médico especialista es el líder del equipo de salud, o el responsable de la oficina de seguridad del paciente o programa de seguridad del paciente, el resto de los miembros del equipo tienen a su cargo los procedimientos de atención y cuidado posoperatorio que según Portocarrero ${ }^{8}$ se encuentran entre las situaciones donde los EA ocurren con mayor frecuencia.

Ahora, por otro lado, en términos de la aplicación existen dos hechos que es importante mencionar para entender si las prácticas están acompañando los planteamientos de seguridad para el paciente. En primera instancia, se encuentra que el 63,74\% de los encuestados perciben que el seguimiento y supervisión de los docentes a los estudiantes durante los procedimientos de aprendizaje se está realizando, pero este porcentaje se puede considerar bajo, teniendo en cuenta la importancia de la supervisión hacia el personal en el proceso de entrenamiento para evitar EA. El otro punto tiene relación con el hecho de que el 69,01\% del personal encuestado reconoce que las normas de seguridad establecidas en los procesos de atención se aplican sin embargo, un 22,68\% afirman que las acciones inseguras pasan desapercibidas y un 15,12\% están indecisos. Estos hechos significan que, a pesar de que existe cierto nivel de adherencia a las normas y guías, no se puede estimar que haya prácticas seguras en el grado necesario que requiere una cultura de seguridad del paciente.

8 PORTOCARREÑO, 2007, Op cit.
Se observa que las creencias del personal pueden ser un elemento que esté afectando el nivel regular de adherencia. En este sentido, a pesar de que el 99,42\% afirman estar de acuerdo o muy de acuerdo en que la seguridad del paciente debe ser un tema obligatorio en las instituciones de salud, es decir, que tienen conciencia de la relevancia del tema, la dificultad parece estar en el factor de los efectos que se perciben frente al reporte, ya que existe un porcentaje considerable de los encuestados que no comunican los eventos adversos o incidentes, debido a posibles medidas disciplinarias, repercusiones ético-legales e incluso por el rechazo de los colegas.

De allí que exista la percepción en el personal de que sus errores son usados en su contra para el 36,41\%, y que el $33,53 \%$ de los encuestados se manifiesten indecisos frente a ello, lo cual evidencia que para los empleados el reporte dentro de la cultura de la seguridad en la institución está mayormente asociado con la prescripción que con un proceso de aprendizaje.

Sin embargo, es importante señalar que en otros aspectos la cultura de la seguridad del paciente se manifiesta con mejores perspectivas. Se reconoce un buen nivel de comunicación y buen ambiente de trabajo, ya que, para tres cuartas partes del personal, la institución promueve un ambiente laboral que favorece la cultura de la seguridad, lo que se expresa en el buen estado de los equipos, en un nivel aceptable de coordinación y cooperación dentro de los servicios y entre ellos, y en procesos administrativos debidamente diseñados y que funcionan. Además, se observa que para la mayoría del personal existe una preocupación de la institución por las prácticas seguras, que se plantea a través de auditoría permanente y procesos de seguimiento y monitoreo.

En este punto en particular se encontró que el 46,79\% del personal reconocen tener una actitud favorable 
frente a la supervisión, lo cual significa que si bien se entiende la necesidad de esta, ya que la mayor parte de los encuestados afirman que la organización desarrolla procesos que garantizan a los usuarios una atención segura, casi la mitad de los empleados plantean no estar de acuerdo con estos procedimientos, lo que vuelve a ratificar lo mencionado anteriormente en relación con una percepción de que la institución tiende a ser punitiva frente a los errores o fallas del personal.

En consecuencia, a pesar de que la institución tenga un sistema de reporte que sirve de herramienta para informar y reportar la ocurrencia de los incidentes y EA, conocido por el 84,38\% de los encuestados, y que se observa en la institución el compromiso y el trabajo para mantenerse actualizada frente a los cambios del entorno con respecto al tema de seguridad del paciente, la política de seguridad del paciente, en relación con los procedimientos y sistema de reporte de incidentes y eventos adversos, no es suficientemente eficaz, debido a que está siendo afectada por la percepción (creencias y actitudes) del personal, bien porque no se aplican de manera regular por miedo, falta de conocimiento (según oficio) o por exceso de trabajo.

Ahora bien, la generación de una cultura de seguridad, por implicar procesos colectivos, requiere esfuerzos permanentes que necesitan tiempo para llegar a que cada persona del equipo de salud tenga conciencia de que la seguridad es prioritaria, y que llegue a plasmarla en todas las prácticas de los servicios de salud que presta. Por esta razón, se puede afirmar que aunque no se llega aún a tener una cultura de seguridad del todo eficaz, en la institución existen indicios y fortalezas que llevan a pensar que están en el camino para llegar a tal objetivo.

De hecho, una fortaleza importante consiste en la baja rotación del personal, sobretodo si se considera que un gran porcentaje del personal de la institución reconoce que la cultura de la seguridad es promovida constantemente, que son continuamente motivados en esta materia y que el sistema de reporte (comunicación y análisis de incidentes) con que cuenta la organización es efectivo. De tal manera, se entiende que para el $70 \%$ de los empleados la cultura de la seguridad influya en los valores, creencias y comportamientos de las personas que trabajan en ella.

Otra fortaleza se encuentra en el convencimiento que tiene casi el 70\% del personal en el hecho de que se da un trato digno a los pacientes y en que la atención es buena, lo que se manifiesta en los altos promedios frente a la importancia de estar y sentirse preparado para resolver rápida y adecuadamente las situaciones de emergencia (alerta situacional), y sobre la necesidad de informar a los pacientes acerca de la finalidad del tratamiento y sobre la asistencia clínica de la que van a ser objeto como formas de atención segura.

En síntesis, el grado de conocimiento del personal de la IPS acerca de legislación y de la cultura de seguridad es bueno; sin embargo, las actitudes frente a la normatividad y a la supervisión tienen un impacto considerable en la cultura de la seguridad, ya que el reporte se ve afectado por la creencia de los empleados en que la institución llega a ser punitiva con quien comete errores. Además, el personal observa fallas en los procesos de coordinación (nivel de influencias organizacionales) que comprometen la eficacia de las prácticas seguras.

Estos elementos marcan dos aspectos para trabajar en la institución, para fortalecer su cultura de seguridad. Por un lado, se requiere aunar esfuerzos institucionales para reducir el miedo o la inseguridad frente al reporte de EA, propiciando la confianza del personal en que este no se va a convertir en causal de rechazo o despido, y en que la supervisión más que un ejercicio de vigilancia es una herramienta de orientación y aprendizaje institucional. Por otro lado, se requiere 
mejorar las estrategias de coordinación dentro y entre los diversos servicios o áreas de atención de la IPS, con el fin de distribuir cargas y optimizar los efectos de la comunicación de EA.

Finalmente, en términos del trabajo de campo, es importante mencionar un aspecto en relación con el instrumento aplicado, que puede calificarse de dificultad. Como se explicó, para el diligenciamiento de la encuesta se utilizó la escala de Likert, dentro de la cual la calificación 3 correspondió con la categoría "Ni de acuerdo ni en desacuerdo". Así, en el análisis de algunos puntos se encontró que esta calificación influyó bajando el porcentaje de la pregunta respondida, generando un alto margen de "indecisos", y, a su vez, afectó los promedios generales por nivel. Así, esta información generó obstáculos en el análisis ya que no queda claro si esta respuesta refleja indiferencia o desconocimiento de los encuestados frente a lo preguntado o si es producto de la misma desconfianza encontrada acerca del reporte. De esta forma, se considera importante hacer la salvedad de que al diseñar una encuesta cuyos resultados midan un aspecto tan importante para una IPS, como es la cultura de seguridad para el paciente, se debe definir de manera más específica, para el encuestado, el rango intermedio con el fin de disminuir las ambigüedades.

\section{RECOMENDACIONES}

Los eventos adversos en los cuidados del paciente tienen unas incidencias económicas, sanitarias y sociales que en el país no se han investigado lo suficiente. Por esta razón, se requiere de esfuerzos investigativos permanentes alrededor de este tema que relaciona la calidad no solo con las prácticas sino con factores éticos y de cultura en las organizaciones.

Los resultados de esta investigación pueden darle orientación a posibles estrategias de la IPS, objeto de estudio, para fortalecer su cultura de la seguridad. En este sentido, realizar este ejercicio investigativo de manera sistemática podría llegar a ser una fuente de mejoras y ajustes pertinentes para sus procesos de calidad.

Respecto a futuros usos de un instrumento para indagar sobre conocimientos y percepción en torno a la cultura de seguridad del paciente, a partir de la herramienta aplicada, se sugiere ajustar la escala Likert en su grado 3, con el fin de evitar ambigüedades en el posterior análisis de los datos, de manera que se puede cambiar a un "no sabe/no responde". Además, señalar a quien va a ser encuestado que trate de contestar las preguntas de manera que solo elija este grado cuando efectivamente no sepa la respuesta.

\section{BIBLIOGRAFÍA}

AGENCIA DE CALIDAD DEL SISTEMA NACIONAL DE SALUD. Declaración de profesionales por la seguridad del paciente. [on line] 2006 [2009-10-08] Recuperado de Internet: http:/ www.aseedar-td.org/docsints/cat_view/39-general.html?dir=ubqswyysqat

AGUIRRE-GAS, H. \& VÁZQUEZ-ESTUPIÑÁN, F. El error médico. Eventos adversos. En: Cirugía y Cirujanos, 2006, vol. 74, no. 6, p. 495-503.

AIBAR REMÓN, Carlos y ARANAZ, Andrés. La seguridad del paciente: Un reto del sistema sanitario y un desafío para la formación. En: Seguridad del paciente y prevención de eventos adversos relacionados con la asistencia sanitaria. Agencia de Calidad del Sistema Nacional de Salud. Ministerio de Sanidad y Consumo. [on line] 2008 [citado 2010-01-04]. Recuperado de Internet: http://82.223.161.224/MSC-Tutoriales/MSCCD1/index.html 
ALIANZA MUNDIAL PARA LA SALUD. Seguridad del paciente. [on line] 2008 [2009-10-25] Recuperado de Internet: http://www.scielo.org.ar/ pdf/aap/v107n5/v107n5a01.pdf.

BARBOSA RAMÍREZ, Lucía; CÁRDENAS, Elizabeth; GARCÍA, Liliana; MORE, Liliana y MUÑZ, María. Establecimiento de una línea de base de la cultura de la seguridad de los pacientes en un hospital universitario en Bogotá. En: Revista Iberoamericana de Psicología: Ciencia y Tecnología. 2008, no. 1, p. 19-28.

BRENNAN, T.A. \& LEAPE L.L. Adverse Events, Negligence in Hospitalized Patients: Results from the Harvard Medical Practice Study. En: Perspect Health Risk Manage, 1991, vol. 11, no. 2, p. 2-8.

CAMPAÑA, G. Errores médicos en el ambiente quirúrgico. Cómo prevenirlos. Parte I. Generalidades. En: Revista Chilena de Cirugía, 2006, vol. 58, no. 3, p. 235-238.

COLES, J. Reporting of Adverse Clinical Incidents: International Views and Experience. London: CASPE Research, 2001.

COLOMBIA. CONGRESO DE LA REPÚBLICA. Ley 10 (1, enero, 1990) Por la cual se reorganiza el Sistema Nacional de Salud y se dictan otras disposiciones. [on line] [citado 2008-09-1] Recuperado de Internet: http://www.dafp.gov.co/leyes/ L0010_90.HTM

COLOMBIA. CONGRESO DE LA REPÚBLICA. Ley 100 (23, diciembre, 1993). Por la cual se crea el Sistema de Seguridad Social integral y se dictan otras disposiciones. [on line] [citado 2007-07-01] Recuperado de Internet: www.secretariasenado. gov.co/.../ley/1993/ley_0100_1993.html
COLOMBIA. CONGRESO DE LA REPÚBLICA. Constitución Política de Colombia. Bogotá: Legis, 1991.

COLOMBIA. DEPARTAMENTO NACIONAL DE PLANEACIÓN. Lineamientos para una política nacional de calidad, documento Compes. Bogotá: Departamento Nacional de Planeación, 2006.

COLOMBIA. MINISTERIO DE SALUD. Decreto 2309 (15, octubre, 2002). Por el cual se define el Sistema Obligatorio de Garantía de Calidad de la Atención de Salud del Sistema General de Seguridad Social en Salud. [on line] [citado 2008-01-08] Recuperado de Internet: http://www.anestesianet. com/normasydecretos/Decreto2309.pdf

COLOMBIA. MINISTERIO DE LA PROTECCIÓN SOCIAL. Resolución 1446 (8, mayo, 2006). Anexo Técnico. Por la cual se define el Sistema de Información para la Calidad y se adoptan los indicadores de monitoría del Sistema Obligatorio de Garantía de Calidad de la Atención en Salud. [on line] [citado 2008-01-08] Recuperado de Internet: http://www.consultorsalud.com/resoluciones.html

COLOMBIA. MINISTERIO DE LA PROTEGCIÓN SOCIAL. Decreto 1011 (3, abril, 2006). Por el cual se establece el Sistema Obligatorio de Garantía de Calidad de la Atención de Salud del Sistema General de Seguridad Social en Salud. [on line] [citado 2009-07-04] Recuperado de Internet: http://www.cruzrojacolombiana.org/normatividad/ otras_leyes/decreto\%201011\%20de\%202006\%20 \%20Calidad.pdf

COLOMBIA. MINISTERIO DE LA PROTECCIÓN SOCIAL. Eventos Adversos dentro del ordenamiento Jurídico. Bogotá: Ministerio de la Protección Social, 2006. 
COLOMBIA. MINISTERIO DE LA PROTECCIÓN SOCIAL. Herramientas para promover la estrategia de la seguridad del paciente en el Sistema Obligatorio de Garantía de la Calidad. Bogotá: Fundación Fitec, 2007.

COLOMBIA. MINISTERIO DE LA PROTEGCIÓN SOCIAL. El Estudio Ibeas y La Políitica de Seguridad del Paciente en Colombia. [on line] 2007 [citado 2008-08-01]. Recuperado de Internet: http://www.minproteccionsocial.gov. co/VBeContent/Library/Documents/DocNewsNo14913DocumentNo4633.pdf

COLOMBIA. MINISTERIO DE LA PROTEGCIÓN SOCIAL. Lineamientos para la implementación de la Política de Seguridad del Paciente. Bogotá D.C.: Ministerio de la Protección Social, 2008.

COLOMBIA. MINISTERIO DE LA PROTEGCIÓN SOCIAL. Informe Ibeas. Sistema de Información para la Calidad. [on line] 2009 [citado 2009-11-23]. Recuperado de Internet: http://www. ocsav.info/ocs_contenidos/BOCS02.pdf

DELGADO GALLEGO, María Eugenia. Actitudes y opiniones de actores implicados en la política participativa del control de la calidad de los servicios de salud en Colombia. En: Universe Psychological, 2007, vol. 6, no. 2, p. 345-358.

DONALDSON, Liam. La seguridad del paciente: "No hacer daño". Perspectivas de Salud. La revista de la Organización Panamericana de la Salud. [on line] 2005, vol. 10, no. 1 [citado 2009-10-08]. Recuperado de Internet: http://www.paho.org/ Spanish/DD/PIN/Numero21_last.htm
EHSANI, J., JACKSON, T. y DUCKETT, S. The Incidence and Cost of Adverse Events in Victorian Hospitals. En: Med J., 2006, no. 184, p. 551-555.

ESANDI, M. et ál. La cultura de la seguridad. En: La Revista del ITAES, vol. 9, no. 1, p. 18-25.

ESPAÑA. MINISTERIO DE SANIDAD Y CONSUMO. Estudio nacional sobre los efectos adversos ligados a la hospitalización ENEAS. Madrid: Ministerio de Sanidad y Consumo, 2006.

ESPAÑA. MINISTERIO DE SANIDAD Y POLÍTICA SOCIAL. Análisis de la cultura sobre seguridad del paciente en el ámbito hospitalario del Sistema Nacional de Salud Español. Madrid: Ministerio de Sanidad y Polííca Social, 2009.

FRANCO, A. La seguridad clínica de los pacientes: entendiendo el problema. En: Revista Colombia Médica, 2005, vol. 36, p. 130-133.

Fundamentos de seguridad al paciente para disminuir errores médicos. Cali: Universidad del Valle, 2006.

El reporte de eventos adversos: motor de la gestión clínica. En: Vía salud, 2007, vol. 38, p. 10-14.

GAITÁN, Hernando et ál. Incidencia y evitabilidad de eventos adversos en pacientes hospitalizados en tres instituciones hospitalarias en Colombia, 2006. En: Revista Salud Pública, 2008, vol. 10, no. 2, p. 215-226.

GIBBONS, M. et ál. The New Production of Knowledge: Science and Research in Contemporary Societies. Barcelona: Ediciones Pomares - Corredor S.A., 1997. 
GIRALDO, M. Calidoscopio una mirada a la calidad de la salud: Colombia a la vanguardia en seguridad del paciente. Bogotá: Ministerio de la Protección Social, 2007.

GONZÁLEZ VILLANUEVA, P. Aproximación al concepto "creencias". [on line] En: Educare21. 2004, no. 5. [citado 2009-10-09] Recuperado de Intenet: http://enfermeria21.com/educare/educare05/ensenando/ensenando2.htm

HELMREICH, Robert L. El factor humano en los accidentes aéreos. En: Revista Investigación y Ciencia, 1997, julio, no. 250.

HOLLANDER, E. Principios y métodos de la psicología social. Buenos Aires: Amorrortu, 1986.

IBÁÑEZ, J. Perspectivas de la investigación social: el diseño en las tres perspectivas. En: GARCÍA FERRANDO, M.; IBÁÑEZ, J. \& ALVIRA, F. (Comps.). El análisis de la realidad social. Métodos y técnicas de investigación social. Madrid: Alianza, 2000.

JAIMOVICH, D. La globalización de la salud y su impacto en la entrega del cuidado a la salud. Seminario Internacional de Calidad en Salud. Medellín: Joint Commission Internacional. 2008, abril, no. 21-22.

KERGUELEN, A. ¿Qué implica hablar de una cultura de seguridad de pacientes? En: Vía Salud, 2007, vol. 41, p. 4.

LONDONOO, J. Modelo para gestionar la seguridad de los pacientes en las instituciones de salud. Medellín: Seminario Seguridad del Paciente, 2008.

LÓPEZ SILVA, S. Errores en medicina. [on line] 2008 [citado 2008-02-25] Recuperado de Inter- net: http://www.jornada.unam.mx/2000/06/26/ cien-errores.html

MEJÍA MARTÍNEZ, G. y PORTOCARRERO, J. Gestión clínica, eventos adversos y responsabilidad civil médica. En: Vía Salud, 2006, no. 32, p. 10-12.

MEJÍA-ORTEGA, L. M. y FRANCO-GIRALDO, A. Protección social y modelos de desarrollo que garanticen la salvaguardia en eventos adversos que pudieran causar detrimento a la salud. En: Revista Salud Pública, 2007, vol. 9, no. 3, p. 471-83.

MOGUEL PARRA, G., AMOR SANTOYO, S. y BARRAGÁN PÉREZ, E. Seguridad de los pacientes, prioridad del Hospital Infantil de México: punto de vista del médico como paciente. En: Boletín Médico del Hospital Infantil de México, vol. 65 , no. 5, p. 407-411.

MORA, J. y FRENK, H. Seguridad del paciente. Curso taller. México: Secretaría de Salud, 2002.

ORGANIZACIÓN PANAMERICANA DE LA SALUD. Política y estrategia regional para la garantía de la calidad de la atención sanitaria, incluyendo la seguridad del paciente. [on line] 2002 [citado 2007-10-05] Recuperado de Internet: <http:// www.ops-oms.org/spanish/gov/csp/csp27.r10-s. pdf $>$.

PAGET, Marianne. The Unity of Mistakes: a Phenomenological Interpretation of Medical Work. Philadelphia: Temple University Press, 1998.

PORTOCARRERO, J. Seguridad del paciente: primero, cultura justa. En: Vía Salud, 2007, no. 39, p. 2-5. 
----------. Seguridad del paciente: indicio de evento adverso medicamentoso. En: Vía Salud, 2007, no. 42, p. 2-10.

RAMOS, Daniel. Diseño de una metodología para la elaboración de diagnósticos institucionales, administrativos y financieros aplicados al desarrollo de la administración pública municipal mexicana. En: Gaceta Mexicana de Administración Pública Estatal y Municipal, 1994, no. 45, 46 y 47, p. 79-98.

REASON, James. Gestión del riesgo de accidentes en las organizaciones. Reino Unido: Ashgate Publishing Limited, 1997.

RECIO SEGOVIANO, M. Herramientas para la gestión y mejora de la seguridad del paciente. Cómo pasar de las ideas a la acción. Lansing: Universidad de Michigan, 2005.

RED IBEROAMERICANA PARA LA ACREDITACIÓN DE LA CALIDAD DE LA EDUCACIÓN SUPERIOR. Glosario. [on line] [citado 2008-11-18] Recuperado de Internet: http://www. riaces.net/glosariom.html.

RODRÍGUEZ, Andrés. Reporte de eventos adversos: experiencia del Instituto de Ortopedia Infantil Roosvelt. En: Vía salud, 2007, no. 42, p. 5-10.
RODRÍGUEZ, Carlos. Requisitos de entrada a la Acreditación en Salud. [on line] 2007 [citado 2009-09-03] Recuperado de Internet: http://www. imbanaco.com/simposioseguridad/memorias_simposio/carlos_rodriguez.pdf

-----------. Hacia una Política Nacional de seguridad del paciente, importancia de la acreditación en salud. [on line] 2008 [citado 2010-03-3] Recuperado de Internet: http://www.acreditacionensalud.org. co/catalogo/docs/REVISTA\%20No\%2072.pdf

SIERRA, H. Eventos adversos en pacientes hospitalizados en tres instituciones centro de gestión hospitalaria (2007). Posición del centro de gestión hospitalaria ante el XVI Foro Internacional Gestión Clínica: Seguridad del Paciente". En: Vía Salud, 2008, vol. 40, p. 2-7.

VANDER ZANDER, J. W. Manual de psicología social. México: Paidós, 1989.

VITOLO, Fabián. La notificación de incidentes, errores y eventos adversos. [on line] 2004 [citado 2009-10-09] Recuperado de Internet: <http:// www.noble-arp.com/src/img_up/27082008.1.pdf> 